\title{
A novel computational method for solving nonlinear Volterra integro-differential equation
}

\author{
Musa Cakir ${ }^{1}$, Baransel Gunes ${ }^{2, *}$, Hakki Duru³ \\ ${ }^{1}$ Dept. of Mathematics, Faculty of Science, Yuzuncu Yil University, Van, Turkey \\ ${ }^{2}$ Dept. of Mathematics, Faculty of Science, Yuzuncu Yil University, Van, Turkey \\ ${ }^{3}$ Dept. of Mathematics, Faculty of Science, Yuzuncu Yil University, Van, Turkey \\ *Corresponding Author: baransel_gunes_91@hotmail.com
}

\begin{abstract}
In this paper, we study quasilinear Volterra integro-differential equations (VIDEs). Asymptotic estimates are made for the solution of VIDE. Finite difference scheme, which is accomplished by the method of integral identities using interpolating quadrature rules with weight functions and remainder term in integral form, is presented for the VIDE. Error estimates are carried out according to the discrete maximum norm. It is given an effective quasilinearization technique for solving nonlinear VIDE. The theoretical results are performed on numerical examples.
\end{abstract}

Keywords: Error bounds; finite difference method; Volterra integro-differential equation.

\section{Introduction}

In this research, we consider the following quasilinear Volterra integro-differential initial value problem:

$$
\begin{array}{r}
L u:=u^{\prime}(t)+f(t, u(t))+\int_{0}^{t} K(t, s, u(s)) d s=0, \\
t \in I=(0, T]
\end{array}
$$

$u(0)=A$,

where $\bar{I}=[0, T]$ and $A$ is a given constant and $f(t, u(t))$ $((t, u) \in \bar{I} \times \mathbb{R})$ and $K(t, s, u(s))((t, s, u) \in \bar{I} \times \bar{I} \times \mathbb{R})$ are given sufficiently smooth functions and moreover

$0<\alpha \leq \frac{\partial f}{\partial u} \leq p^{*}<\infty$.

Volterra integro-differential equations (VIDEs) are typical mathematical models in many areas of science. The solution of VIDEs has been identified as important in physics, engineering, chemistry and biology.

For instance, Kirchoff's second law is considered as follows:

$L \frac{d}{d t} I(t)+R I(t)+\frac{1}{C} \int_{0}^{t} I(\tau) d \tau=E(t)$

where $I(t)$ is the current function, $R$ is the resistance, $L$ is the inductance and $C$ is the capacitance (Zill \& Warren, 2013). On the other hand, Wilson-Cowan model describes the dynamics of interactions between populations:

$$
\begin{aligned}
& \frac{\partial E}{\partial t}=-E+\int W_{e e}\left(x-x^{\prime}\right) f\left(E-\theta_{1}\right) d x \\
& -\int W_{i e}\left(x-x^{\prime}\right) f\left(E-\theta_{2}\right) d x+\psi_{1}(x, t) \\
& \tau \frac{\partial E}{\partial t}=-I+\int W_{e i}\left(x-x^{\prime}\right) f\left(E-\theta_{1}\right) d x \\
& -\int W_{i i}\left(x-x^{\prime}\right) f\left(E-\theta_{2}\right) d x+\psi_{2}(x, t)
\end{aligned}
$$

where initial conditions $E(x, 0)=E_{0}(x), I(x, 0)=I_{0}(x)$ and $x \in \mathbb{R}, t>0 . E(x, t)$ and $I(x, t)$ represent the activity of a population of excitatory and inhibitory neurons, respectively. $W_{i j}$ describes the strength of connection $\theta_{1}$, $\theta_{2}$ and $\tau$ are positive parameters. $\psi_{1}$ and $\psi_{2}$ are external inputs (Singh et al., 2018).

For numerical solutions of these equations, numerous methods have been presented by some authors. Adomian decomposition method (Biazar et al., 2003; Goghary et al., 2005; Wazwaz, 2010), Galerkin method (Maleknejad \& Kojani, 2004), collocation method (Aguilar \& Brunner, 1988; Laib et al., 2018), iterative and non-iterative methods (Ramos, 2009), homotopy perturbation method (Yusufoglu, 2007), Tau method (Mahmoud et al., 2005) and variational iteration method (Wang \& He, 2007) are among these. Recently, the studies of these problems have become significant and have been investigated with different properties. Singularly perturbed VIDEs are considered by many authors. An exponentially fitted 
difference scheme is constructed for these equations (Amiraliyev \& Sevgin, 2006). For singularly perturbed VIDEs with smooth kernel, the coupled method (LDGCFEM) is used (Tao \& Zhang, 2019). The difference scheme and its convergence properties are examined for singularly perturbed VIDEs on a graded mesh (Sevgin, 2014). Moreover, more different studies are seen. Finite difference method is examined for first-order VIDEs (Cimen, 2018). In addition, for high-order linear VIDEs, a new numerical method with Taylor polynomials is developed (Laib et al., 2018). Considering more varied methods, implicit Runge-Kutta method is applied for approximate solution of VIDEs (Brunner, 1984). A computational method is developed for numerical solution of a nonlinear VIDE of fractional order (Saeedi \& Mohseni, 2011). By using Euler polynomials, VIDEs of pantograph-delay type are examined (Mirzaee et al., 2016) and nonlinear Volterra delay integro differential equations have been studied (Gan, 2007). Besides, for high-order integro differential equations with weakly singular kernel, a Galerkin-like method is used (Yüzbaş1 \& Karaçayır, 2016).

In this study, our purpose is to present finite difference method, which is founded by the method of integral identities using basis functions and interpolating quadrature rules with weight and remainder term in integral form. This method is first applied to nonlinear VIDEs. Unlike other methods, our method yields more accurate results for these equations.

Notations. Throughout the paper, $C$ and $C_{0}$ are generic positive constants that are independent of the mesh parameter. For any continuous function $v(x)$ defined on the corresponding interval, we use the maximum norm $\|v\|_{\infty}=\max _{[0, T]}|v(x)|$.

\section{Continuous problem}

In this section, we give some asymptotic behavior of the exact solution, which is needed in the analysis of numerical method.

Lemma 1. Let $p(t), q(t) \in C(\bar{I})$ and $G(t, s) \in C(\bar{I} \times \bar{I})$. Then for the solution $u$ of the problem (1)-(2), the following estimates hold:

$\|u\|_{\infty} \leq C_{0}$

and

$\left\|u^{\prime}\right\|_{\infty} \leq C$ where

$C_{0}=\left(|A|+\alpha^{-1}\|q\|_{\infty}\right) e^{\alpha^{-1} \bar{G} T}$

$C=\|q\|_{\infty}+C_{0}\left(p^{*}+\bar{G} T\right)$

and

$\bar{G}=\max _{I \times I}|G(t, s)|$.

Proof. Applying the mean value theorem to functions in Equation (1), we get

$f(t, u)=f(t, 0)+\frac{\partial f(t, \bar{u})}{\partial u}, \quad \bar{u}=\gamma u, \quad 0<\gamma<1$,

$K(t, s, u(s))=K(t, s, 0)+\frac{\partial}{\partial u} K(t, s, \dot{u}), \quad \dot{u}=\theta u, \quad 0<\theta<1$,

$u^{\prime}(t)+f(t, 0)+\frac{\partial f(t, \bar{u})}{\partial u} u+\int_{0}^{t} \frac{\partial}{\partial u} K(t, s, \dot{u}) u(t) d s$

$+\int_{0}^{t} K(t, s, 0) d s=0$

$u^{\prime}(t)+\frac{\partial f(t, \bar{u})}{\partial u}+\int_{0}^{t} \frac{\partial}{\partial u} K(t, s, \dot{u}) u(t) d s=-f(t, 0)$

$-\int_{0}^{t} K(t, s, 0) d s$.

Then, we can write Equation (1) in the form

$u^{\prime}(t)+p(t) u(t)+\int_{0}^{t} G(t, s) u(s) d s=q(t), t \in I$,

where

$p(t)=\frac{\partial}{\partial u} f(t, \bar{u})$,

$G(t, s)=\frac{\partial}{\partial u} K(t, s, \dot{u})$

and

$q(t)=-f(t, 0)-\int_{0}^{t} K(t, s, 0) d s$.

From (5), we have

$u(t)=u(0) e^{-\int_{0}^{t} p(\eta) d \eta}$

$+\int_{0}^{t}\left[q(s)+\int_{0}^{s} G(s, \xi) u(\xi) d \xi\right] e^{-\int_{s}^{t} p(\eta) d \eta} d s$.

From here, we take

$|u(t)| \leq|A| e^{-\alpha t}$

$+\int_{0}^{t}\left[q(s)+\int_{0}^{s}|G(s, \xi)||u(\xi)| d \xi\right] e^{-\alpha(t-s)} d s$

$\leq|A| e^{-\alpha t}+\alpha^{-1}\|q\|_{\infty}\left(1-e^{-\alpha t}\right)$

$+\alpha^{-1} \bar{G}\left(1-e^{-\alpha t}\right) \int_{0}^{t}|u(\xi)| d \xi$. 
Applying Gronwall's inequality to inequality (6), we obtain

$|u(t)| \leq\left(|A|+\alpha^{-1}\|q\|_{\infty}\right) e^{\alpha^{-1} \bar{G} T}$,

which leads to (3).

Now, we prove inequality (4). From Equation (5), we take

$\left|u^{\prime}(t)\right| \leq|p(t)||u(t)|+|q(t)|$

$+\int_{0}^{t}|G(t, s) \| u(s)| d s$

$\leq p^{*} C_{0}+\|q\|_{\infty}+\bar{G} C_{0} \int_{0}^{t} d s$

$\leq\|q\|_{\infty}+C_{0}\left(p^{*}+\bar{G} T\right)$

which immediately leads to (4).

\section{Discretization and mesh}

$\varpi_{\tau}=\left\{t_{0}<t_{1}<t_{2}<\cdots<t_{N-1}<t_{N}\right\}$

is a non-uniform mesh to a set of discrete points. $t_{i}$ points are called node points and $\tau_{i}=t_{i}-t_{i-1}$ is a meshsize. Before describing our numerical method, we define some notation for the mesh functions. For any mesh function $v(x)$ defined on $\varpi_{\tau}$, we use

$v_{i}=v\left(t_{i}\right), \quad v_{\bar{t}, i}=\frac{v_{i}-v_{i-1}}{\tau}$,

$\|v\|_{\infty}=\|v\|_{\infty, \varpi_{\tau}}=\max _{0 \leq i \leq N}\left|v_{i}\right|$.

To generate the difference method, we begin the following integral identity

$\tau_{i}^{-1} \int_{t_{i-1}}^{t_{i}} L u d t=0$

$\tau_{i}^{-1} \int_{t_{i-1}}^{t_{i}} u^{\prime}(t) \varphi_{i}(t) d t+\tau_{i}^{-1} \int_{t_{i-1}}^{t_{i}} f(t, u) \varphi_{i}(t) d t$

$+\tau_{i}^{-1} \int_{t_{i-1}}^{t_{i}} \int_{0}^{t} K(t, s, u(s)) \varphi_{i}(t) d s d t=0$.

Now, we separately evaluate each term of Equation (7). For $\varphi_{i}(t)=1$, we use interpolating quadrature rules in Amiraliyev \& Mamedov (1995) for each term. For the first term on the right side of Equation (7), we get

$\tau_{i}{ }^{-1} \int_{t_{i-1}}^{t_{i}} u^{\prime}(t) \varphi_{i}(t) d t=u_{\bar{t}, i}+R_{i}{ }^{*}$,

where the truncation error is
$R_{i}^{(*)}=-\int_{t_{i-1}}^{t_{i}} d t \varphi_{i}^{\prime}(t) \int_{t_{i-1}}^{t_{i}} \frac{d u(\xi)}{d \xi} T_{0}(t-\xi) d \xi=0$.

For the second term on the right side of Equation (7), we have

$\tau_{i}^{-1} \int_{t_{i-1}}^{t_{i}} f(t, u) d t=f\left(t_{i}, u_{i}\right)+R_{i}^{(1)}$,

where

$R_{i}^{(1)}=-\tau_{i}^{-1} \int_{t_{i-1}}^{t_{i}}\left(\xi-t_{i-1}\right) \frac{d}{d \xi} f(\xi, u(\xi)) d \xi$.

For the third term on the right side of Equation (7), we obtain

$\tau_{i}^{-1} \int_{t_{i-1}}^{t_{i}} \int_{0}^{t} K(t, s, u(s)) d s d t$

$=\tau_{i}^{-1} \int_{t_{i-1}}^{t_{i}} d t \int_{0}^{t_{i}} K\left(t_{i}, s, u(s)\right) d s$

$=\int_{0}^{t_{i}} K\left(t_{i}, s, u(s)\right) d s+R_{i}^{(2)}$,

where

$R_{i}^{(2)}=-\tau_{i}{ }^{-1} \int_{t_{i-1}}^{t_{i}}\left(\xi-t_{i-1}\right) \frac{d}{d \xi}\left(\int_{0}^{t} K(\xi, s, u(s) d s) d \xi\right.$.

Using the right side rectangle rule for integral term involving kernel function, we have

$\int_{0}^{t_{i}} K\left(t_{i}, s, u(s)\right) d s=\sum_{j=1}^{i} \tau_{j} K_{i, j}\left(t_{i}, t_{j}, u_{j}\right)+R_{i}^{(3)}$,

where

$R_{i}^{(3)}=\sum_{j=1}^{i} \int_{t_{i-1}}^{t_{i}}\left(\xi-t_{j-1}\right) \frac{d}{d \xi} K\left(t_{i}, \xi, u(\xi)\right) d \xi$.

Then, we can write the following difference problem for approximation of VIDE:

$u_{\bar{t}, i}+f\left(t_{i}, u_{i}\right)+\sum_{j=1}^{i} \tau_{j} K_{i, j}\left(t_{i}, t_{j}, u_{j}\right)+R_{i}=0$,

$i=1,2, \ldots, N$,

$u(0)=A$,

where

$R_{i}=R_{i}^{(1)}+R_{i}^{(2)}+R_{i}^{(3)}$.

Thus, by neglecting $R_{i}$, we obtain the difference scheme in the following form: 
$y_{\bar{t}, i}+f\left(t_{i}, y_{i}\right)+\sum_{j=1}^{i} \tau_{j} K_{i, j}\left(t_{i}, t_{j}, y_{j}\right)=0$,

$i=1,2, \ldots, N$,

$y_{0}=A$

\section{Error estimates}

For the investigation of the uniform convergence of this method, let $u_{i}$ be the solution of the problem (1)-(2) and let $y_{i}$ be the solution of the problem (14)-(15). Error function $z_{i}=y_{i}-u_{i} i=0,1, \ldots, N$ is the solution of the following discrete problem:

$l z_{i}=z_{\bar{t}, i}+\left[f\left(t_{i}, y_{i}\right)-f\left(t_{i}, u_{i}\right)\right]$

$+\sum_{j=1}^{i} \tau_{j}\left[K_{i, j}\left(t_{i}, t_{j}, y_{j}\right)-K_{i, j}\left(t_{i}, t_{j}, u_{j}\right)\right]=R_{i}$,

$z_{0}=0$

where $R_{i}$ is given by Equation (13).

Lemma 2. Let $z_{i}$ be the solution of (16)-(17). Then, the estimate

$\|z\|_{\infty, \varpi_{N}} \leq C\|R\|_{\infty, \omega_{N}}$

holds.

Proof. Using the mean value theorem for the functions in Equation (16), we take

$z_{\bar{t}, i}+p_{i} z_{i}+\sum_{j=1}^{i} \tau_{j} \frac{\partial \widetilde{K}}{\partial u} z_{j}=R_{i}$

where

$p_{i}=\frac{\partial f}{\partial u}\left(t_{i}, \overline{\gamma_{l}}\right), \quad \bar{\gamma}_{l}=\gamma \bar{y}_{l}, \quad 0<\gamma<1$.

$y_{i}$ are intermediate points called for by the mean value theorem. From here, we have

$\left|z_{i}\right| \leq \alpha^{-1}\|R\|_{\infty, \omega_{N}}+\alpha^{-1} \widetilde{K} \sum_{j=1}^{i}\left|z_{j}\right|$,

$i=1,2, \ldots, N$.

Applying the difference analogue of Gronwall's inequality to (19), we obtain

$\left|z_{i}\right| \leq \alpha^{-1} e^{\alpha^{-1} \widetilde{K} t_{i}}\|R\|_{\infty, \omega_{N},} \quad i=1,2, \ldots, N$,

where

$\widetilde{K}=\left|\frac{\partial \widetilde{K}}{\partial u}\right|$,

which implies the validity of (18).

Lemma 3. Assume that the mesh function $g_{i}$ is the solution of initial value problem $l_{h} g_{i}:=g_{\bar{t}, i}+a_{i} g_{i}=F_{i}, \quad i=1,2 \ldots, N$,

$g_{0}=A$,

with $\left|F_{i}\right| \leq \mathcal{F}_{i}$ and $\mathcal{F}_{i}$ is a nondecreasing function. Then for the solution of (20)-(21), the following inequality holds:

$\left|g_{i}\right| \leq|A|+\alpha^{-1} \mathcal{F}_{i}, \quad i=1,2 \ldots, N$

Proof. First, we note that for the difference operator $l_{h} g_{i}$ the maximum principle holds in the form: If any mesh function $l_{h} g_{i} \geq 0, i=1,2 \ldots, N$ and $g_{0} \geq 0$, then $g_{i} \geq 0, i=1,2 \ldots, N$. Since $\mathcal{F}_{i}$ is a nondecreasing

$\mathcal{F}_{\bar{t}, i}=\frac{\mathcal{F}_{i}-\mathcal{F}_{i-1}}{\tau} \geq 0$,

the barrier functions can be written in the following form:

$\psi_{i}^{ \pm}= \pm g_{i}+|A|+\alpha^{-1} \mathcal{F}_{i}$

It follows that

$\psi_{0}{ }^{ \pm}= \pm A+|A|+\alpha^{-1} \mathcal{F}_{i} \geq 0$,

$l_{h} \psi_{i}^{ \pm}= \pm \mathcal{F}_{i}+a_{i}|A|+\alpha^{-1} \mathcal{F}_{i} \geq F_{i}+\mathcal{F}_{i} \geq 0$

Thereby, according to the maximum principle, $\psi_{i}^{ \pm} \geq 0$, which proves (22).

Lemma 4. Under the condition of Lemma 1, for the remainder term $R_{i}$ of scheme (14)-(15), the following estimate satisfies

$\|R\|_{\infty, \omega_{N}} \leq C \tau$.

Proof. Now, we separately evaluate the remainder terms in Equation (13). First, we prove Equation (8). From the relation Equation (8) and taking into account Lemma 1, we obtain

$\left|R_{i}^{(1)}\right| \leq \tau_{i}^{-1} \int_{t_{i-1}}^{t_{i}}\left(\xi-t_{i-1}\right)\left|\frac{d}{d \xi} f(\xi, u(\xi))\right| d \xi$

$\leq \tau_{i}^{-1} \int_{t_{i-1}}^{t_{i}}\left(\xi-t_{i-1}\right)\left[\left|\frac{\partial}{\partial \xi} f(\xi, u(\xi))\right|\right.$

$\left.+\left|\frac{\partial}{\partial u} f(\xi, u(\xi))\right|\left|u^{\prime}(\xi)\right|\right] d \xi$

$\leq C \tau_{i}{ }^{-1} \int_{t_{i-1}}^{t_{i}}\left(\xi-t_{i-1}\right)\left|1+u^{\prime}(\xi)\right| d \xi$ 
$\leq C\left\{\tau_{i}+\int_{t_{i-1}}^{t_{i}}\left|u^{\prime}(\xi)\right| d \xi\right\}$

$\leq C \tau_{i,} \quad i=1,2, \ldots, N$.

For Equation (9), we have

$\left|R_{i}^{(2)}\right| \leq \tau_{i}^{-1} \int_{t_{i-1}}^{t_{i}}\left|\left(\xi-t_{i-1}\right) \frac{d}{d \xi}\left(\int_{0}^{t} K(\xi, s, u(s)) d s\right)\right| d \xi$

$\leq \tau_{i}^{-1} \int_{t_{i-1}}^{t_{i}}\left(\xi-t_{i-1}\right)[|K(\xi, t, u(t))|$

$\left.+\int_{0}^{t}\left|\frac{\partial}{\partial \xi} K(\xi, s, u(s)) d s\right|\right] d \xi$

$\leq C \tau_{i}, \quad i=1,2, \ldots, N$.

Next, from Equation (10), we get

$\left|R_{i}{ }^{(3)}\right|=\sum_{j=1}^{i} \int_{t_{i-1}}^{t_{i}}\left(\xi-t_{j-1}\right)\left|\frac{d}{d \xi} K\left(t_{i}, \xi, u(\xi)\right)\right| d \xi$

$\leq \sum_{j=1}^{i} \int_{t_{i-1}}^{t_{i}}\left(\xi-t_{j-1}\right)\left[\left|\frac{\partial}{\partial \xi} K\left(t_{i}, \xi, u(\xi)\right)\right|\right.$

$\left.+\left|\frac{\partial}{\partial u} K\left(t_{i}, \xi, u(\xi)\right)\right|\left|u^{\prime}(\xi)\right|\right] d \xi$

$\leq C \tau_{i}, i=1,2, \ldots, N$

Finally, substituting the inequalities (24), (25) and (26) in Equation (13), we obtain inequality (18).

From the two previous lemmas, we immediately obtain the main result of the following paper.

Theorem 1. Let $u(x)$ be the solution of the problem (1)(2) and let $y$ be solution of the approximate problem (14)(15). Then the following estimate satisfies

$\|y-u\|_{\infty, \omega_{\tau}} \leq C \tau_{i}$.

Proof. Let $z_{i}=y_{i}-u_{i} i=0,1, \ldots, N$. Then, from the discrete problem (16)-(17) we can rewrite it in the form

$z_{\bar{t}, i}+p_{i} z_{i}+\sum_{j=1}^{i} \tau_{j} \frac{\partial \widetilde{K}}{\partial u} z_{j}=R_{i}$

$i=0,1, \ldots, N$,

$z_{0}=0$

After applying Lemma 3 to the discrete problem (28)(29), we get $\left|z_{i}\right| \leq \alpha^{-1}\|R\|_{\infty, \omega_{N}}+\alpha^{-1} \widetilde{K} \sum_{j=1}^{i}\left|z_{j}\right|, i=0,1, \ldots, N$

From here, by using the difference analogue of Gronwall's inequality, we obtain the following inequality:

$\left|z_{i}\right| \leq \alpha^{-1} e^{\alpha^{-1} \widetilde{K} T}\|R\|_{\infty, \omega_{N}}$

$\leq C\|R\|_{\infty, \omega_{N}}, \quad i=1,2, \ldots, N$.

Further, from Lemma 4, we see that

$\|R\|_{\infty, \omega_{N}} \leq C \tau$

From the inequalities (30) and (31), we arrive at (27).

\section{Algorithm and numerical results}

In this section, we present the numerical results for difference scheme (14)-(15). Because of the nonlinear term, we use the quasilinearization technique. Applying this technique to difference scheme (14)-(15), we obtain

$y_{\bar{t}, i}^{(n)}+f\left(t_{i}, y_{i}^{(n-1)}\right)+\frac{\partial}{\partial y} f\left(t_{i}, y_{i}^{(n-1)}\right)\left(y_{i}^{(n)}-y_{i}^{(n-1)}\right)$

$+\sum_{j=1}^{i} \tau_{j}\left[K_{i j}\left(t_{i}, t_{j}, y_{j}^{(n-1)}\right)\right.$

$\left.+\frac{\partial}{\partial y} K_{i j}\left(t_{i}, t_{j}, y_{j}^{(n-1)}\right)\left(\left(y_{j}^{(n)}-y_{j}^{(n-1)}\right)\right)\right]=0$,

$y_{0}^{(n)}=A, \quad i=1,2, \ldots, N$

If the elimination method is taken into consideration, we have

$y_{i}^{(n)}=\frac{A_{i} y_{i-1}{ }^{(n)}+B_{i} y_{i}^{(n-1)}-C_{i}-\widetilde{K}_{l}}{\frac{1}{\tau_{i}}+\frac{\partial}{\partial y} f\left(t_{i}, y_{i}^{(n-1)}\right)+\tau_{i} \frac{\partial}{\partial y} K_{i j}\left(t_{i}, t_{i}, y_{i}^{(n-1)}\right)}$,

$y_{0}^{(n)}=A$,

where

$A_{i}=\frac{1}{\tau_{i}^{\prime}}$

$B_{i}=\frac{\partial}{\partial y} f\left(t_{i}, y_{i}^{(n-1)}\right)+\tau_{i} \frac{\partial}{\partial y} K_{i j}\left(t_{i}, t_{i}, y_{i}^{(n-1)}\right)$,

$C_{i}=f\left(t_{i}, y_{i}^{(n-1)}\right)+\tau_{i} K_{i j}\left(t_{i}, t_{i}, y_{i}^{(n-1)}\right)$,

$\widetilde{K}_{l}=\sum_{j=1}^{i-1} \tau_{j} K_{i j}\left(t_{i}, t_{j}, y_{j}^{(n-1)}\right)$. 
The stopping criterion is

$$
\max _{i}\left|y_{i}^{(n)}-y_{i}^{(n-1)}\right| \leq 10^{-3}
$$

and $y_{i}{ }^{(0)}$ is the initial process. Then, the difference scheme is tested on the following examples. Exact errors are determined as

$e^{N}=\left|y_{i}-u_{i}\right|$,

where $u_{i}$ is the exact solution and $y_{i}$ is the approximate solution.
Example 1. We consider the following nonlinear Volterra integro-differential equation:

$u^{\prime}(t)+\frac{u^{2}(t)}{2}+u(t)-\frac{1}{2}+\int_{0}^{t} u^{2}(s) d s=0$,

with $u(0)=1$. The exact solution of the equation is $u(t)=e^{-t}$. The computational results are presented in Table 1. In addition, the obtained results from Table 1 are shown in Figure 1.

Table 1. Error approximations for Example 1.

\begin{tabular}{cccc}
\hline $\boldsymbol{t}_{\boldsymbol{i}}$ & $\boldsymbol{y}_{\boldsymbol{i}}$ & $\boldsymbol{u}_{\boldsymbol{i}}$ & $\boldsymbol{y}_{\boldsymbol{i}}-\boldsymbol{u}_{\boldsymbol{i}}$ \\
\hline 0.000 & 1.000000000 & 1.000000000 & 0.000000000 \\
0.125 & 0.882496902 & 0.879922493 & 0.002574410 \\
0.250 & 0.778800783 & 0.778748879 & 0.000051904 \\
0.375 & 0.687289278 & 0.694229264 & 0.006939985 \\
0.500 & 0.606530659 & 0.623990032 & 0.017459372 \\
0.625 & 0.535261428 & 0.565788861 & 0.030527432 \\
0.750 & 0.472366552 & 0.517629831 & 0.045263278 \\
0.875 & 0.416862019 & 0.477799240 & 0.060937220 \\
1.000 & 0.367879441 & 0.444850916 & 0.076971475 \\
\hline
\end{tabular}

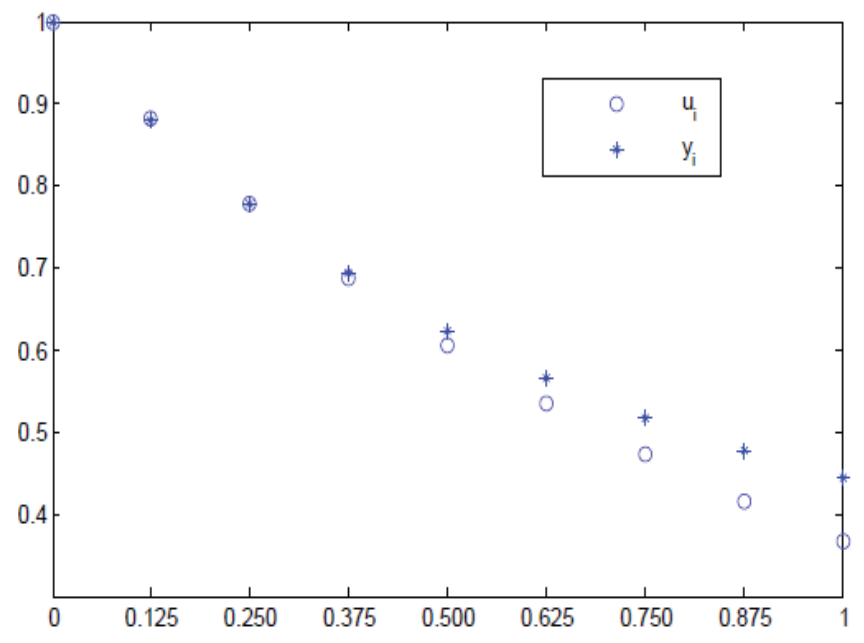

Fig. 1. Comparison of $u_{i}$ and $y_{i}$ for Example 1.

Example 2. We take into account another nonlinear Volterra integro-differential equation:

$u^{\prime}(t)+e^{u}+u(t)+\int_{0}^{t} \tanh (u(s)) d s=0, u(0)=1$.
The exact solution of this equation is unknown. Thus, we use the double-mesh principle to estimate the errors and compute the experimental rates of convergence in the computed solution. That is, we compare the computed solution with the solution on a mesh that is twice as fine (Amiraliyev \& Duru, 2005; Cakir \& Amiraliyev, 2007). The error estimates are denoted by

$e^{N}=\max _{i}\left|y_{i}^{N}-y_{i}^{2 N}\right|$.

The convergence rates are calculated as follows:

$p^{N}=\frac{\ln \left(e^{N} / e^{2 N}\right)}{\ln 2}$.

Error approximations and convergence rates for Example (2) are given in Tables 2-3.

Error approximations which are obtained from Tables 2-3 are shown in Figures 2-3 for some values of $N$. 
Table 2. Error approximations and convergence rates for different values of $N$.

\begin{tabular}{ccccc}
\hline & $\boldsymbol{N}=\mathbf{8}$ & $\boldsymbol{N}=\mathbf{1 6}$ & $\boldsymbol{N}=\mathbf{3 2}$ & $\boldsymbol{N}=\mathbf{6 4}$ \\
\hline$r_{0}$ & 0.02970507 & 0.01615199 & 0.00845788 & 0.00433473 \\
$r_{1}$ & 0.01608326 & 0.00845792 & 0.00433440 & 0.00219515 \\
$p$ & 0.88514899 & 0.93333706 & 0.96446294 & 0.98162238 \\
\hline
\end{tabular}

Table 3. Error approximations and convergence rates for different values of $N$.

\begin{tabular}{ccccc}
\hline & $\boldsymbol{N}=\mathbf{1 2 8}$ & $\boldsymbol{N}=\mathbf{2 5 6}$ & $\boldsymbol{N}=\mathbf{5 1 2}$ & $\boldsymbol{N}=\mathbf{1 0 2 4}$ \\
\hline$r_{0}$ & 0.00219515 & 0.00110470 & 0.00055415 & 0.00027753 \\
$r_{1}$ & 0.00110470 & 0.00055415 & 0.00027753 & 0.00013888 \\
$p$ & 0.99065946 & 0.99530439 & 0.99763936 & 0.99881619 \\
\hline
\end{tabular}

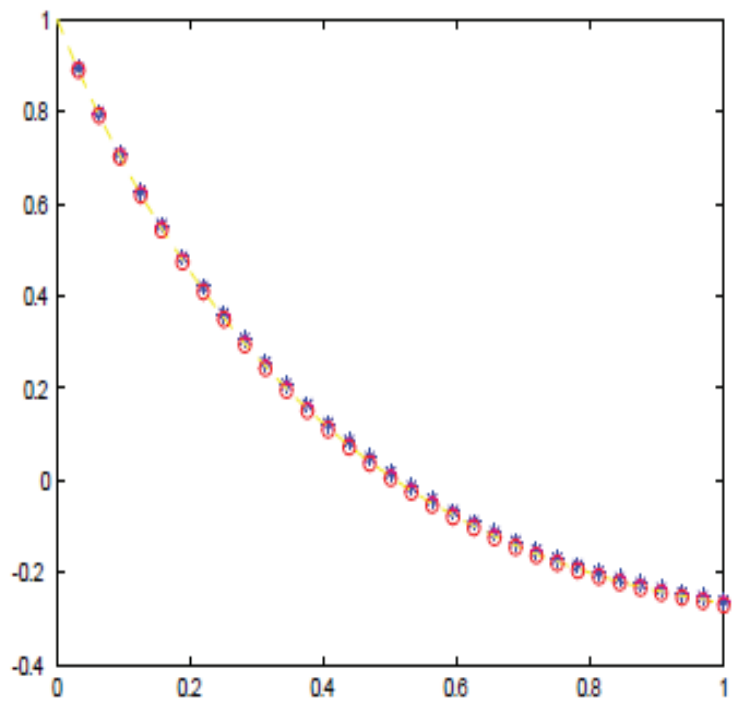

Fig. 2. Error approximations for $N=32$.

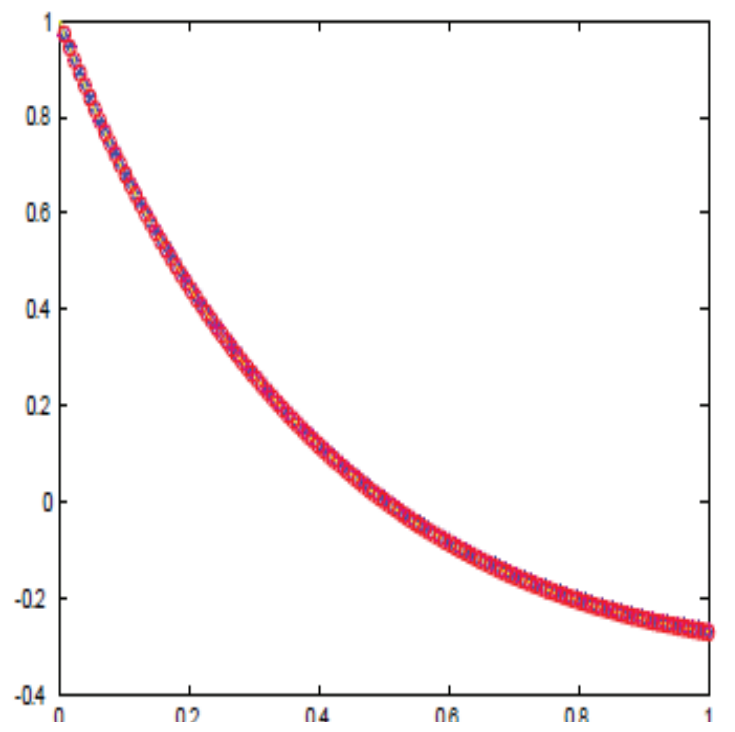

Fig. 3. Error approximations for $N=128$.
Example 3. We tackle the following initial value problem:

$u^{\prime}(t)+\cosh (u(t))+\int_{0}^{t} \sin (u(s)) d s=0, u(0)=1$.
The exact solution of this equation is unknown. Using the double mesh principle, we find experimental rates of convergence. The computational results are shown in Table 4.

Table 4. Error approximations and convergence rates for different values of $N$.

\begin{tabular}{ccccc}
\hline & $\boldsymbol{N}=\mathbf{1 2 8}$ & $\boldsymbol{N}=\mathbf{2 5 6}$ & $\boldsymbol{N}=\mathbf{5 1 2}$ & $\boldsymbol{N}=\mathbf{1 0 2 4}$ \\
\hline$r_{0}$ & 0.00038189 & 0.00019341 & 0.00009733 & 0.00004882 \\
$r_{1}$ & 0.00019342 & 0.00009733 & 0.00004882 & 0.00002445 \\
$p$ & 0.98140912 & 0.99074969 & 0.99534816 & 0.99765610 \\
\hline
\end{tabular}




\section{Discussion and Conclusion}

In this study, we have presented a new and effective numerical method for the quasilinear Volterra integrodifferential equations. The stability of the solution for continuous problem was examined by using the maximum principle, and the difference schemes were taken according to the discrete maximum norm. In the numerical algorithm, the quasilinear technique was used because of nonlinear terms. The theoretical results were tested on samples. It is shown from the tables that the numerical results confirm the theoretical results. Numerical investigations can be carried out for the various types such as delay and singularly perturbed equations with different physical properties.

\section{References}

Amiraliyev, G.M. \& Duru, H. (2005). A note on a parametrized singular perturbation problem. Journal of Computational and Applied Mathematics, 182: 233-242.

Amiraliyev, G.M. \& Mamedov, Y.D. (1995). Difference schemes on the uniform mesh for singularly perturbed pseudo-parabolic equations. Tr. J. of Math., 19: 207-222.

Amiraliyev, G.M. \& Şevgin, S. (2006). Uniform difference method for singularly perturbed Volterraintegro differential equations. Applied Mathematics and Computation, 179: 731-741.

Aguilar, M. \& Brunner, H. (1988). Collocation method for second-order Volterra integro-differential equations. Applied Numerical Mathematics, 4: 455-470.

Biazar, J., Babolian, E. \& Islam, R. (2003). Solution of a system of Volterra integral equations of the first kind by Adomian method. Appl. Math. Comput., 139: 249-258.

Brunner, H. (1984). Implicit Runge-Kutta methods of optimal order for Volterra integro-differential equations. Math. Comp., 42: 95-109.

Cakir, M. \& Amiraliyev, G.M. (2007). Numerical solution of a singularly perturbed three-point boundary value problem. International Journal of Computer Mathematics, 84: 1465-1481.

Cimen, E. (2018). A computational method for Volterra integro-differential equations. Journal of Science and Technology, 11(3): 347-352.

Gan, S. (2007). Dissipativity of $\theta$-methods for nonlinear
Volterra delay-integro-differential equations. Journal of Computational and Applied Mathematics, 206: 898-907.

Goghary, H.S., Javadi, S. \& Babolian, E. (2005). Restarted Adomian method for system of nonlinear Volterra integral equations. Appl. Math. Comput., 161: 745-751.

Laib, H., Bellour, A. \& Bousselsal, M. (2018). Numerical solution of high-order linear Volterra integro-differential equations by using Taylor collocation method. International Journal of Computer Mathematics, 96: 1066-1085.

Mahmoud, J.P., Rahimi-Ardabili, M.Y. \& Shahmorad, S. (2005). Numerical solution of Volterra integrodifferential equations by the Tau method with the Chebyshev and Legendre bases. Applied Mathematics and Computation, 170: 314-338.

Makroglu, A. (1983). A Block-by-block method for the numerical solution of Volterra delay integro-differential equations. Computing, 30: 49-62.

Maleknejad, K. \& Kojani, M. T. (2004). Solving linear integro-differential equation system by Galerkin methods with hybrid functions. Appl. Math. Comput., 159: 603612.

Mirzaee, F., Bimesl, S. \& Tohidi, E. (2016). A numerical framework for solving high-order-pantograph delay Volterra integro-differential equations. Kuwait J. Sci. 43(1): 69-83.

Ramos, J.J. (2009). Iterative and non-iterative methods for nonlinear Volterra integro-differential equations. Applied Mathematics and Computation, 214: 287-296.

Saeedi, H. \& Mohseni, M.M. (2011). Numerical solution of nonlinear Volterra integro-differential equations of arbitrary order by CAS wavelets. Communications in Nonlinear Science and Numerical Simulation, 16: 12161226.

Singh, C.S., Singh, A.K. \& Sahoo, J.K. (2018). Numerical scheme based on operational matrices for integro-differential equations. International Journal of Engineering \& Technology, 7(4.41): 50-54.

Sevgin, S. (2014). Numerical solution of a singularly perturbed Volterra integro-differential equations. Advances in Difference Equations a Springer Open Journal, 171.

Tao, X. \& Zhang, Y. (2019). The coupled method for singularly perturbed Volterra integro-differential 
equations. Advances in Difference Equations a Springer Open Journal, 217.

Wang, S.Q. \& He, J.H. (2007). Variational iteration method for solving integro-differential equations. Physics Letters A 367: 188-191.

Wazwaz, A.M. (2010). The combined Laplace transformAdomian decomposition method for handling nonlinear Volterra integro-differential equation. Linear and Nonlinear Integral Equations, 216: 1304-1309.

Yusufoglu, E. (2007). An efficient algorithm for solving integro-differential equations system. Appl. Math. Comput., 192: 51-55.

Yüzbaşı, Ş. \& Karaçayır, M. (2016). A Galerkin-like approach to solve high-order integro-differential equations with weakly singular kernel. Kuwait J. Sci. 43(2): 106120.

Zill, D. G. \& Warren, S. W. (2013). Differential equations with boundary value problems. Section 7.4: Operational Properties II. Pp. 305. Brooks/ Cole Cengage Learning.

Submitted : 19/03/2020

Revised : 28/04/2020

Accepted : 12/08/2020

DOI $\quad: 10.48129 /$ kjs.v48i1.9386 